\title{
INTEGRAÇÃO SAÚDE E EDUCAÇÃO COM O USO DAS TIC NO PROCESSO DE FORMAÇÃO DE PROFESSORES EM SERVIÇO
}

\author{
Ana Kelly de Lima e SILVA ${ }^{1}$ \\ Flaviana dos Santos SILVA ${ }^{2}$ \\ Carla Cristina ROMANO ${ }^{3}$
}

\begin{abstract}
RESUMO: Este artigo tem o propósito de descrever o processo de formação em serviço de professores de ensino médio em uma escola estadual no município de Ilhéus na tentativa de integrar "saúde-educação" iniciado em março/2009. A pesquisa tem o apoio da Fundação de Amparo a Pesquisa do Estado da Bahia - FAPESB, e o objetivo é o conscientizar os alunos, professores e comunidade acerca dos temas como vulnerabilidade dos jovens às DST/Aids, gravidez precoce, dependência química e suas conseqüências, alimentação saudável, dentre outros. Para tanto, adotou-se como mídias pedagógicas as Tecnologias de Informação e Comunicação - TIC no desenvolvimento das atividades propostas aos alunos. As intervenções têm sido realizadas semanalmente na escola em dois momentos: nas reuniões pedagógicas e Sala de Aula (S.A.); e na Universidade Estadual de Santa Cruz - UESC, nos Laboratórios de Parasitologia e Zoologia de Vertebrados, favorecendo a construção do conhecimento dos conteúdos curriculares da disciplina de Biologia. Como resultados das atividades realizadas neste processo de formação em serviço, os alunos e professores puderam desenvolver com praticidade e criatividade os temas propostos. Neste sentido, foi possível observar a recontextualização e a integração das diferentes mídias aos conteúdos curriculares e ao contexto da comunidade escolar como um todo, contando, inclusive com a participação dos gestores, dando vida às teorias educacionais na realidade da escola.
\end{abstract}

PALAVRAS-CHAVE: Tecnologias de Informação e Comunicação - TIC. Ensino médio. Formação de professores. Trabalho educativo. Integração saúde - educação.

\section{Introdução}

As primeiras iniciativas de uso das tecnologias de informação e comunicação (TIC) na educação brasileira tiveram início da década 80 (TAVARES, 1997), e a partir dai houve um grande movimento para formação de professores com o fim de prepará-los para inserirem essas tecnologias em benefício da aprendizagem e no desenvolvimento de diferentes habilidades nos alunos.

\footnotetext{
${ }^{1}$ UESC - Universidade Estadual de Santa Cruz. Departamento de Ciências Biológicas. Ilhéus - BA - Brasil. 45662-900 - anakellybmd@gmail.com

${ }^{2}$ UESC - Universidade Estadual de Santa Cruz. Departamento de Ciências Exatas e Tecnológicas. Ilhéus - BA Brasil.45662-900 - flavianadss@gmail.com

${ }^{3}$ UESC - Universidade Estadual de Santa Cruz.Departamento de Ciências Biológicas. Ilhéus - BA - Brasil. 45662-900 - romanocc@uol.com.br
} 
O advento das TIC no processo educacional justifica-se porque propicia que escolas tenham acesso ao “[...] conhecimento compartilhado, acelere as mudanças necessárias, agilize as trocas entre alunos, professores e instituições [...]” (MORAN, 2009) para transcender barreiras e dificuldades, almejando um ensino de qualidade.

Na tentativa de se obter melhoramentos no ensino entende-se que é importe que os alunos que têm acessos à escola e permanecem nesta, ao final de sua formação educacional, adquira habilidades para exercer sua cidadania plena.

Segundo Saviani (1980), a Escola deve fazer a mediação entre “o senso comum e a consciência filosófica” (crítica), possibilitando ao educando desenvolver uma visão científica do mundo que o cerca.

Esta mediação será feita pelo conhecimento desenvolvido na escola ao longo do ensino fundamental até início do ensino médio previsto no currículo escolar. As atividades estarão centradas na comunicação e elaboração de novos conhecimentos vinculados ao saber universal e significativo para a vida do homem brasileiro contemporâneo (SAVIANI, 1980).

Para atender este fim, a formação dos professores se fundamentará no esquema da “reflexão na ação” e “reflexão sobre ação”, mediada por diálogos, discussões e acompanhamentos constantes como unidade básica para o desenvolvimento profissional e contribuir com a qualidade de ensino para atingir o objetivo principal da educação que é ajudar a tornar as pessoas mais livres e menos dependentes do poder econômico, político e social (IMBERNÓN, 2001).

Esta abordagem de formação é adequada quando se busca realizar a formação de professores no contexto escolar com o fim prepará-los para inserirem em sua prática o uso das tecnologias da informação e comunicação, em especial das mídias pedagógicas ${ }^{4}$ na educação.

Com este propósito, o presente trabalho tem como objetivo principal descrever o processo de formação em serviço de professores do Ensino Médio para a integração saúdeeducação com suporte nas TIC, de escolas de ensino médio de Ilhéus - Bahia para favorecer a aprendizagem de conceitos de Biologia, tendo como apoio a Fundação de Amparo a Pesquisa do Estado da Bahia - FAPESB.

\section{Desenvolvimento e contexto do trabalho}

\footnotetext{
${ }^{4}$ Mídia pedagógica “é um dispositivo técnico-semiótico (um sistema semiótico de representações e um dispositivo tecnológico)” (PERAYA, 1997). Trata-se de instrumentos que permitam o acesso e produção de informações e conhecimentos. Neste artigo, consideram-se como mídias pedagógicas: o computador em conjunto com os softwares educacionais, aplicativos como editores de texto, a rede Internet, ou qualquer outro equipamento tecnológico que potencializa a construção ou produção do aluno.
} 
Para dar início às atividades do projeto de pesquisa foram selecionadas duas escolas pertencentes ao município Ilhéus. Os diretores das escolas foram contatados por telefone e agendou-se uma reunião com o propósito de lhes apresentar ao projeto e solicitar que o convite fosse estendido aos professores da escola. Após o aceite, foi entregue a cada diretor um questionário de articulação de todas as ações que seriam desenvolvidas durante o trabalho.

Para dar andamento às tarefas, cinco professores foram consultados e preencheram o Termo de Consentimento Livre e Esclarecido, previamente aprovado pelo comitê de Ética e Pesquisa da Universidade Estadual de Santa Cruz (UESC).

Dando continuidade à primeira etapa do projeto, cada professor participante respondeu a um questionário diagnóstico com perguntas como: formação acadêmica, tempo de formação, anos de magistérios e há quanto tempo ministra a disciplina.

$\mathrm{Na}$ etapa seguinte, buscou-se também verificar quais temas deveriam ser tratados inicialmente e quais seriam definidos como prioritários no contexto vivenciado pelos professores. Tais escolhas foram registradas por meio de uma carta de apresentação. Os professores das escolas definiram as prioridades do estudo, numa escala de 0 a 7 (sendo 0 o menos relevante e 7 o mais relevante).

As estratégias usadas na formação de professores para inserirem esses temas com atividades que despertem o interesse e que promovessem a aprendizagem contextualizada e significativa ocorreram a partir do acompanhamento no ambiente de trabalho por parte dos integrantes da equipe do projeto de pesquisa.

Nas reuniões pedagógicas realizadas entre professores e diretores da escola foram definidas dinâmicas que seriam utilizadas por cada professor de acordo com cada tema que seria trabalhado em sala de aula (S.A) previsto no cronograma de assuntos previamente estabelecidos pelas escolas. Em cada encontro foram apresentadas e aplicadas como atividades complementares aos professores e alunos da primeira e segunda série de ensino médio, novas estratégias de metodologias de trabalho para a integração do conhecimento abordado em S.A ao conhecimento das TIC.

Para tanto, foram aplicados questionários aos professores com o propósito de avaliar o nível de conhecimento sobre as TIC, bem como sua utilização como mídias pedagógicas no processo de ensino. 
O conhecimento dos alunos inicialmente também foi avaliado a partir da aplicação de dois tipos de questionários referentes aos temas Mitose ${ }^{5}$ e Drogas Ilícitas, o que nos permitiu verificar com maior precisão a aplicabilidade destes assuntos.

Com o propósito de permitir diferentes reflexões aos professores, estes, foram auxiliados nas intervenções para associar os conteúdos curriculares propostos nos planos de ensino da disciplina envolvida a atividades desafiadoras e que permitiriam a participação ativa dos alunos.

Neste processo a reflexão na ação foi realizada no momento em que o professor desenvolvia seu trabalho com os alunos (SCHON, 2000). Na reflexão sobre a ação o professor teve um pensamento que ocorreu retrospectivamente sobre uma situação vivenciada anteriormente (ZEICHNER, 1993).

Os alunos e professores foram então convidados a participar de atividades práticas realizadas nos laboratórios de Parasitologia Humana e Zoologia de Vertebrados que ficam na Universidade Estadual de Santa Cruz - UESC.

Um dos temas abordados com os alunos da $1^{\text {a }}$ série de ensino médio (21 ao total) de uma das escolas de Ilhéus - Bahia foi a observação da Mitose em células do ápice radicular da cebola.

Assim, os professores foram orientados a expor em S.A o tema buscando a conceituação e apresentação das fases. Articulado a aula teórica, ocorreu uma prática que consistia na observação de lâminas utilizando o Microscópio óptico com o intuito de visualizar as quatro fases da mitose (prófase, metáfase, anáfase e telófase).

Para introduzir o tema na prática foram passados dois filmes que explicavam as quatro fases da Mitose. Em seguida, os alunos foram orientados a produzirem as lâminas para a visualização no microscópio. As Figuras 1, 2, 3, e 4 ilustram o processo para obtenção das lâminas e sua posterior observação das diferentes fases da mitose observada ao microscópio.

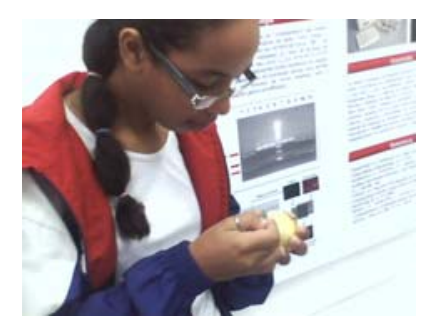

Figura 1 - Corte da cebola para a preparação da lâmina.

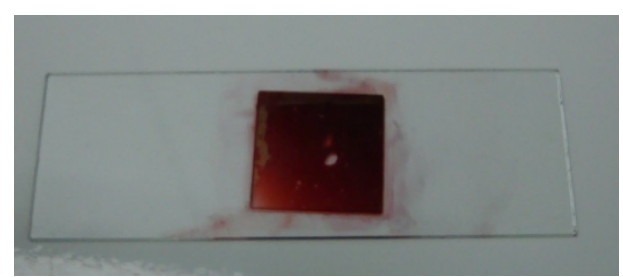

Figura 2 - Lâmina pronta para ser levada ao microscópio.

\footnotetext{
${ }^{5}$ Mitose é o processo pelo qual as células eucarióticas dividem seus cromossomas entre duas células filhas. Este processo é dividido em quatro fases: prófase, metáfase, anáfase e telófase.
} 


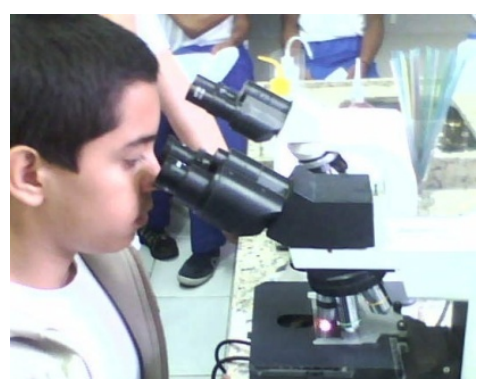

Figura 3 - Visualização da lâmina utilizando-se microscópio óptico.

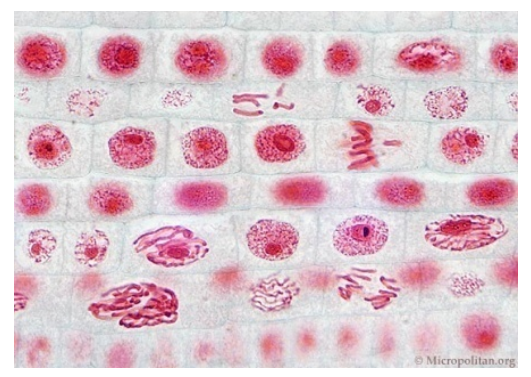

Figura 4 - Apresentação das diferentes fases ao microscópio. ${ }^{6}$

Com os alunos da $2^{\mathrm{a}}$ série de ensino médio foi proposto o tema Drogas Ilícitas. A atividade proposta visou complementar o que já havia sido trabalhado pelo professor como assunto de aula. Na S.A foram trabalhados os conceitos, problemas e aplicações referentes às drogas enquanto que na atividade que seria desenvolvida posteriormente pelos alunos, utilizaram-se filmes como auxilio para o desenvolvimento do tema.

Os alunos foram orientados a produzirem folders e vídeos com o propósito de sensibilizá-los sobre a não utilização das drogas. Para que o trabalho fosse realizado, foi entregue um roteiro que continham orientações para a confecção do folder que seria preenchido pelos escolares da segunda série de ensino médio, a partir da busca de informações sobre o tema, utilizando-se as TIC. Os alunos, por meio das ferramentas de busca da internet, deveriam ainda escolher um programa que serviria de aplicativo para a produção do panfleto informativo.

Após a entrega e avaliação da produção dos alunos, o melhor trabalho foi escolhido para que fossem confeccionadas cópias que seriam entregues aos colegas, professores, funcionários da escola e à comunidade vizinha.

\footnotetext{
${ }^{6}$ Consulte The Botanical Guarden (2008).
} 
As Figuras 5 e 6 ilustram os trabalhos realizados pelos alunos com apoio das mídias pedagógicas.

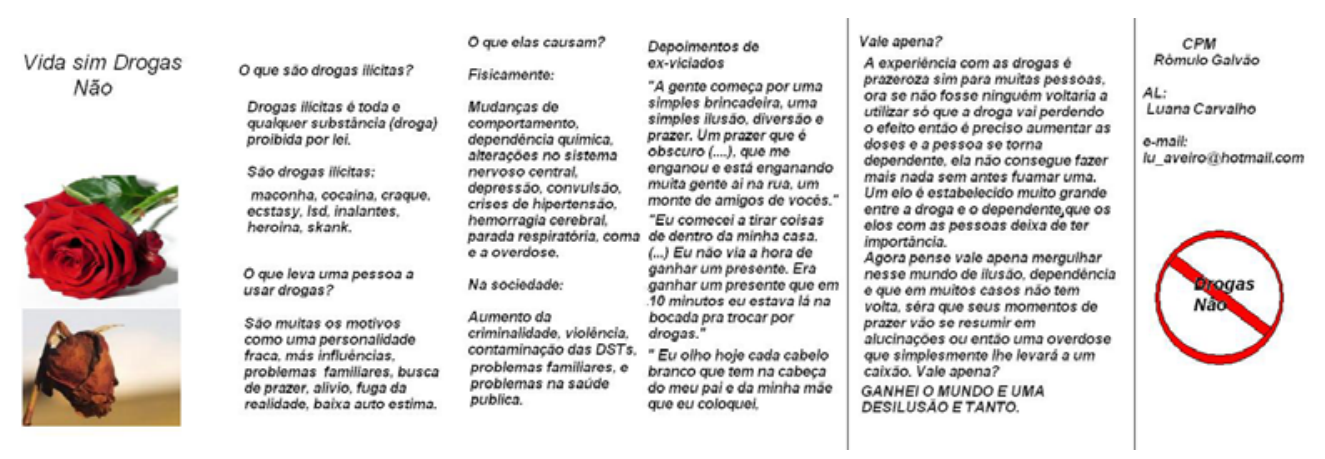

Figura 5 - Visualização folder.

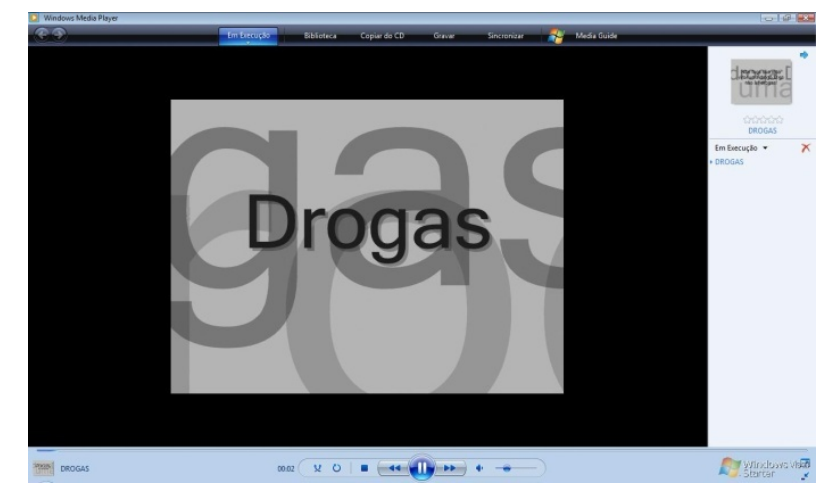

Figura 7 - Visualização do filme.?

Com base nas atividades realizadas no processo de formação, observamos grande participação dos alunos e professores, que serão apresentados e discutidos na próxima seção.

\section{Resultados e considerações}

No processo de formação em serviço dos professores observamos que é importante considerar o contexto de atuação e buscar estratégias que permitam reflexões em diferentes níveis.

Neste trabalho consideremos que a forma de atuar do professor é um fator fundamental, pois oferece condições para que os alunos avancem no seu próprio processo de aprender (SILVA, F.; SILVA, M.; SCHLÜNZEN, 2007).

\footnotetext{
${ }^{7}$ Consulte Drogas (2008).
} 
A primeira prática realizada indicou que os alunos puderam construir conhecimentos acerca do processo de MITOSE e pode ainda ser visto melhoramento na forma de compreender o processo acerca de sua importância para a vida.

Tal fato pode ser verificado na aplicação de um questionário realizado que avaliou o nível de conhecimento dos alunos. Os gráficos 1 e 2 a seguir apresentam o que os alunos conheciam sobre o tema antes e depois respectivamente, do processo de intervenção com os professores.

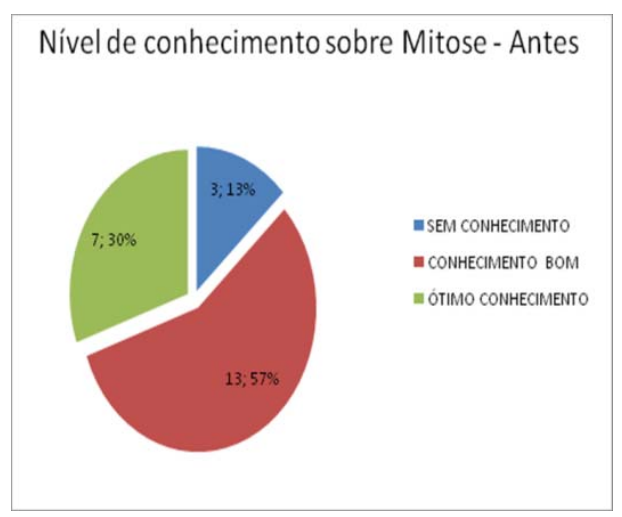

Gráfico 1 - Nível de conhecimento dos alunos antes de realizada a prática de mitose.

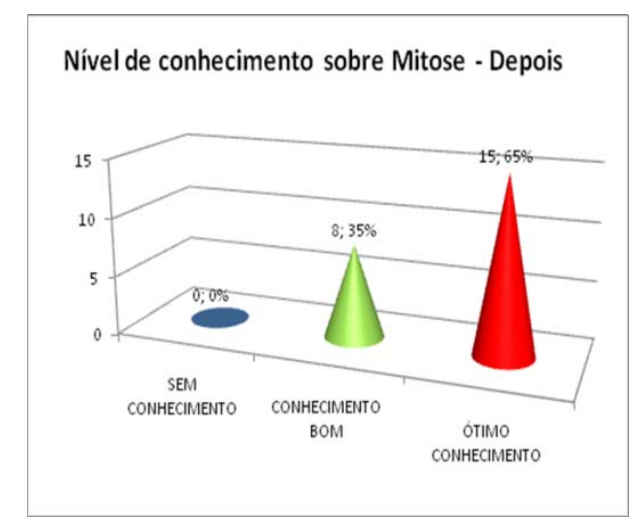

Gráfico 2 - Nível de conhecimento dos alunos após realizada a prática de mitose.

Os resultados observados a partir dos gráficos 1 e 2 demonstraram que os alunos que inicialmente não apresentavam nenhum conhecimento sobre o assunto, após realizado a aula relataram uma mudança no seu nível de conhecimento que pode ser observado a partir do relato abaixo:

[...] até o momento não sabia muitas coisas, mas depois dessa prática pude aprimorar meus conhecimentos sobre mitose. (V.15 anos) 
Para os alunos que inicialmente demonstravam um nível de conhecimento avaliado como “bom”, pode-se observar que estes ao fim da prática, já apresentavam mudanças, passando do nível de conhecimento "bom”, ao “ótimo”,

Na avaliação direcionada à formação dos professores em serviço, os gráficos 3, 4, 5 e 6 demonstram respectivamente o comportamento dos educadores frente ao conhecimento das TIC, sua freqüência na utilização e os tipos de aplicativos que servem de apoio à rotina praticada em S.A.

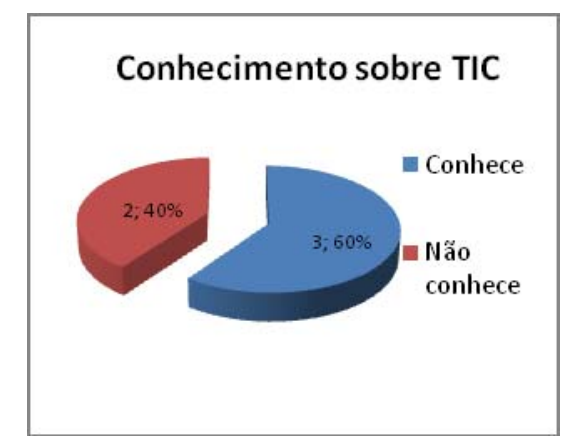

Gráfico 3 - Apresenta o conhecimento dos professores sobre as TIC.

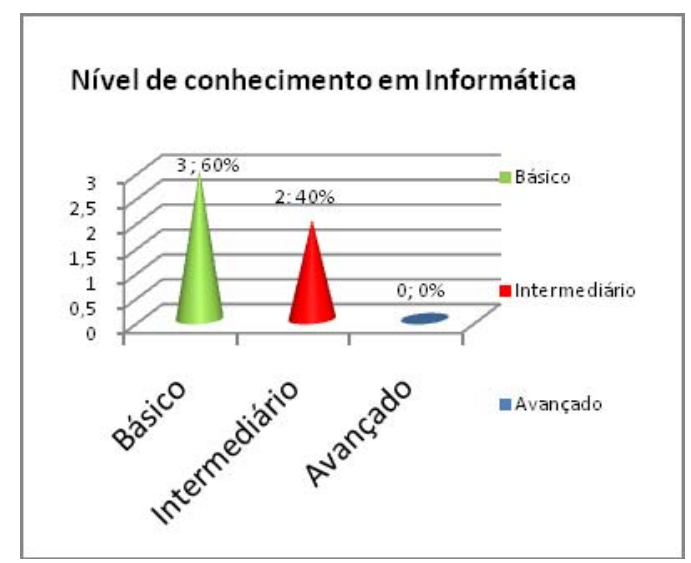

Gráfico 4 - Nível de conhecimento dos professores em Informática.

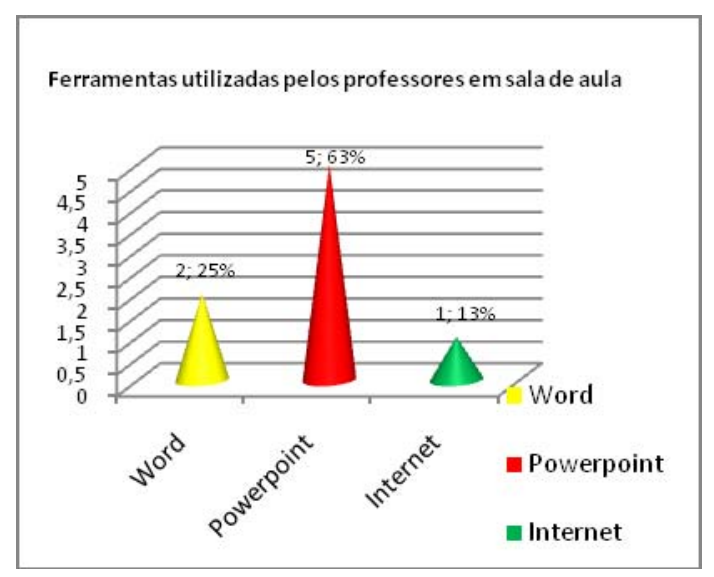

Gráfico 5 - Ferramentas utilizadas pelos professores. 
Diante das análises sobre os gráficos, foi possível observar que 60\% dos professores entrevistados sabiam o significado da palavra “TIC”, entretanto não souberam exemplificar ferramentas que poderiam ser aplicadas em atividades relacionadas com os conteúdos. (gráfico 3).

Baseado destas constatações foi importante orientá-los a propor atividades a partir de seus conhecimentos sobre as TIC. Além disso, desvendar quais aplicativos os alunos conheciam e quais dificuldades poderiam enfrentar durante a produção.

Nas intervenções realizadas (confecção do panfleto e vídeo), os aplicativos utilizados estão apresentados no gráfico 6.

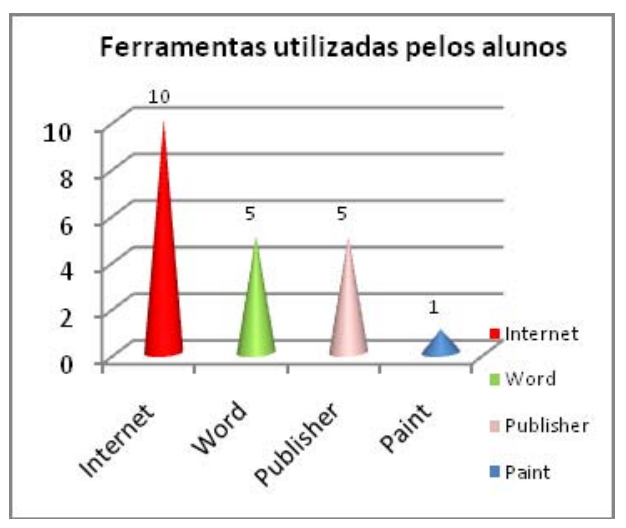

Gráfico 6 - Ferramentas utilizadas pelos alunos na realização da atividade proposta.

Com estes aplicativos os alunos desenvolveram com praticidade e criatividade a atividade proposta. Além disso, sistematizaram seus conhecimentos sobre o tema abordado, além de possibilitar a exposição de opiniões a partir da criação de panfletos e da aula prática sobre Mitose.

Diante das atividades desenvolvidas neste processo de formação em serviço, foi possível criar um ambiente reflexivo, pois os professores passaram a compreender que ao trabalhar com as estratégias apresentadas, atuaram como facilitadores, incentivadores, desafiadores e investigadores de sua própria prática pedagógica e da aprendizagem individual e em grupo (ALMEIDA, 2001).

Neste sentido, foi possível observar a recontextualização e a integração das diferentes mídias aos conteúdos curriculares e ao contexto da comunidade escolar como um todo, 
contando, inclusive com a participação dos gestores, dando vida às teorias educacionais na realidade da escola.

A partir do exposto, acreditamos que a formação dos professores para integrar o tema saúde-educação neste projeto na medida do possível tem sido realizada com êxito. Porém esperamos a definição de políticas públicas para que as estratégias utilizadas neste processo de formação sejam ampliadas para um número maior de professores.

\section{INFORMATION AND COMMUNICATION TECHNOLOGY (TIC) IN A HEALTH- EDUCATION PROGRAM FOR TEACHERS}

ABSTRACT: This paper describes teachers in-service development process in a Ilhéus high school an attempt to integrate "health-education program started in March the year 2009. The research is supported by Fundação de Amparo a Pesquisa do Estado da Bahia FAPESB, and the goal is to educate students, teachers and community about issues such as vulnerability of young people to DST/AIDS, teenage pregnancy, substance abuse and its consequences, eating, among others. Therefore, the Information and Communication Technology (TIC) was used as pedagogical media in the development of activities offered to students. The interventions have been held weekly at the school on two occasions: in meetings and educational classroom (S.A) and the State University of Santa Cruz - UESC, Laboratories of Parasitology and Zoology of Vertebrates, favoring the knowledge of curriculum content the discipline of biology. As a result of the activities developed in this inservice development process, students and teachers were able to develop creativity and practicality with the proposed themes. In this sense, we could observe the re-contextualization and integration of differents medias to curriculum content and context of the school community as a whole, counting, including the participation of managers, giving life to the educational theories on the reality of school.

KEYWORDS: Information and communication technology (TIC). Teachers in-service development process. High School in Bahia State. 


\section{REFERÊNCIAS}

ALMEIDA, M. E. B. Educação, projetos, tecnologia e conhecimento. São Paulo: PROEM, 2001.

DROGAS. Disponível em: <http://www.youtube.com/watch?v=aLgG3wcJloI>. Acesso em: 11 jun. 2008.

IMBERNÓN, F. Formação docente e profissional: formar-se para mudança e a incerteza. 2. ed. Tradução de Silvana Cobucci Leite. São Paulo: Cortez, 2001.

MORAN, J. M. Novos desafios na educação: a internet na educação presencial e virtual. Disponível em: <www.eca.usp.br/prof/moran/novos.htm>. Acesso em: 07 jun. 2008.

PERAYA, D. As formas de comunicação pedagógica “midiatizada”: o socioeducativo e o didático. 1997. Disponível em: <http://www.scielo.br/pdf/es/v18n59/18n59a03.pdf>. Acesso em: 16 jun. 2006.

SAVIANI, D. Educação: do senso comum à consciência filosófica. São Paulo: Cortez, 1980.

SCHÖN, D. A. Educando o profissional reflexivo: um novo design para o ensino e aprendizagem. Tradução de Roberto Cataldo Costa. Porto Alegre: Artes Médicas Sul, 2000.

SILVA, F. S.; SILVA, M. G. M.; SCHLÜNZEN, E. T. M. Formação para a mudança: criando um ambiente reflexivo para o uso das mídias pedagógicas no processo educacional In:

CONFERÊNCIA INTERNACIONAL DE TECNOLOGIAS DE INFORMAÇÃO E COMUNICAÇÃO NA EDUCAÇÃO, 5., 2007, Minho. Disponível em:

$<$ http://www.nonio.uminho.pt/documentos/actas/actchal2007/107.pdf >. Acesso em: 07 jun. 2008.

TAVARES, N. R. B. História da informática educacional no Brasil observado a partir de três projetos públicos. 1997. Disponível em:

<http://quimica.fe.usp.br/textos/tics/ticspdf/neide.pdf>. Acesso em: 07 jun. 2008.

THE BOTANICAL GUARDEN. Disponível em: < http://www.microscopyuk.org.uk/micropolitan/botany/mitosis_onion.jpg>. Acesso em: 11 jun. 2008.

ZEICHNER, K. A formação de professores: idéias e práticas. Lisboa: Educa, 1993. 